\title{
Understanding Visceral Ischaemia in Acute Type B Dissection: State of the Art, Challenges and Future Perspectives
}

\author{
T. Donati, A. Patel, and M. Sallam
}

\subsection{Visceral Malperfusion}

\subsubsection{Definition}

Visceral malperfusion is an ill-defined entity; in the available literature, it is variably used to refer to mesenteric and renal malperfusion or to mesenteric malperfusion alone with or without end-organ ischaemia. This distinction is crucial to allow clarity of discussion and avoid misinterpretation or misuse of available data from the literature.

It is our opinion that when referring to visceral malperfusion in the context of aortic dissection, this has to refer to the clinical scenario related to malperfusion of the visceral branches of the aorta.

According to Henry Gray's textbook of anatomy [1], the visceral branches of the abdominal aorta include the coeliac axis, the superior mesenteric, the inferior mesenteric, the middle suprarenals, the renals, the internal spermatic and the ovarian arteries.

Within the limits of our knowledge, only involvement of the superior mesenteric and renal arteries (with the occasional exception of the coeliac axis) is related to relevant clinical scenarios in the clinical setting and therefore can be the

T. Donati $(\bowtie) \cdot$ A. Patel $\cdot$ M. Sallam

Guy's and St Thomas' NHS Foundation Trust,

London, UK

e-mail: tommaso.donati@gstt.nhs.uk object of a meaningful discussion; however other entities like MP and ischaemia of the adrenal gland might be clinically relevant and wrongly overlooked.

\subsubsection{The Relevance of VMP in the Setting of Type A and B Dissection and Why We Cannot Ignore Type A Dissections}

The incidence of visceral malperfusion differs between type A and type B reflecting somehow the different populations of patients affected by these very closely related but at the same time different conditions [2-4].

Distal extension of a type A dissection to the aorta and its branches is an independent marker of a severity [5, 6]. In-hospital mortality for patients included in the IRAD registry presenting with type A dissections complicated by mesenteric malperfusion undergoing surgical or hybrid treatment has been reported to be $41.7 \%[7,8]$.

Although strictly speaking beyond the aim of this chapter, we believe it is also important to highlight the relevance of visceral malperfusion in setting of acute type A dissection.

There are multiple reasons for it:

- Visceral malperfusion (with particular mention to mesenteric malperfusion) in the setting 
of acute type A dissection is a leading cause and independent predictor factor for in-hospital mortality; distal extension of a type A dissection to the aorta and its branches is an independent marker of a severity [5, 6, 9]. In-hospital mortality for patients included in the IRAD registry presenting with type A dissections complicated by mesenteric malperfusion undergoing surgical or hybrid treatment has been reported to be as high as $41.7 \%[7,8]$.

- Recognition of this complication is often delayed as a consequence of the predominant role given to emergency repair of the ascending aorta and the difficult assessment of signs and symptoms in the immediate postoperative period.

- There is increasing interest for a staged approach for acute type A dissection complicated by visceral malperfusion advocating treatment of the malperfusion first followed by surgical repair of the ascending aorta (hence in a certain way questioning the dogma of the neat separation between type A and type B in terms of timing and strategy of treatment) [2, 10, 11].

- Even after successful repair of the ascending aorta, type A dissection with distal progression to the descending thoracic and visceral aorta can present exactly the same challenges of a type $\mathrm{B}$ dissection and in the long term can result in chronic "type B" dissection.

- Given the current strategies for treatment of VMP, a better communication and a better integration between different teams (cardiothoracic, vascular and endovascular interventionist) is likely beneficial for patients for a multidisciplinary approach aiming at early detection and treatment of reversible cause of a clinically relevant VMP.

\subsubsection{Mechanisms of Obstruction Reviewed}

Williams and his collaborators in a paper published in 1997 have described the mechanisms involved in the obstruction of the visceral branches of the aorta for the first time. They described two main mechanisms that are still accepted today, one "dynamic" and one "static" [12].

Although further mechanisms have been proposed [13] mainly as a combination of dynamic and static components, ultimately the mainand most relevant-difference between the two mechanisms is the direct involvement or not of the ostia of the aortic branch in consideration.

A dynamic obstruction refers mainly to the reduction of flow to a given artery due to a haemodynamic conflict between the true and the false lumen in the aorta. Dynamic obstructions can be responsible of clinical pictures of intermittent malperfusion; however it is also important to clarify that "dynamic" does not necessarily translate with "intermittent" (Fig. 30.1).

Static obstructions on the other hand are characterised by a direct involvement of the ostia of the aortic branch with possible progression of the dissection into the branch and a possible conflict between the true and the false lumen within the aortic branch.

The involvement of a branch can result in different scenarios of which the most common are:

- Progression of the false lumen into a branch with distal re-entry and maintained perfusion of vessel through the false, or both the true and false, lumen (Fig. 30.2)

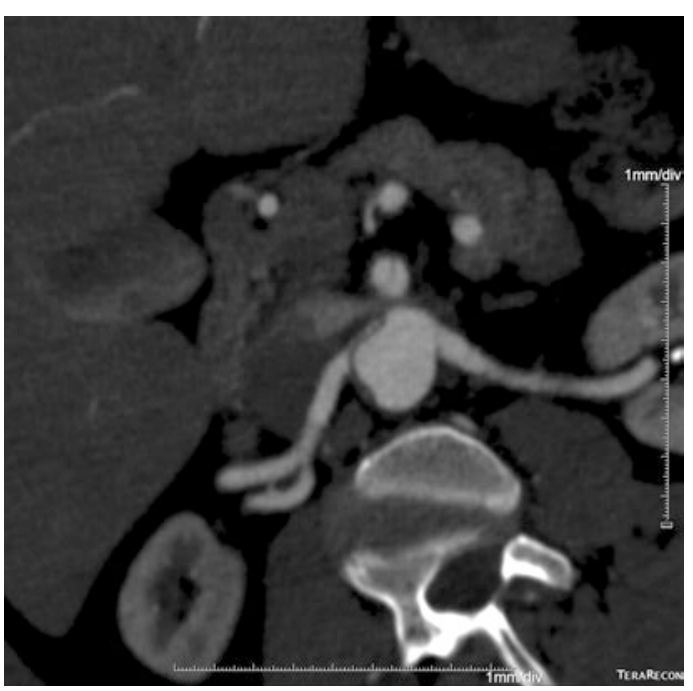

Fig. 30.1 Dynamic obstruction of the right renal artery with delayed perfusion but no obvious malperfusion 


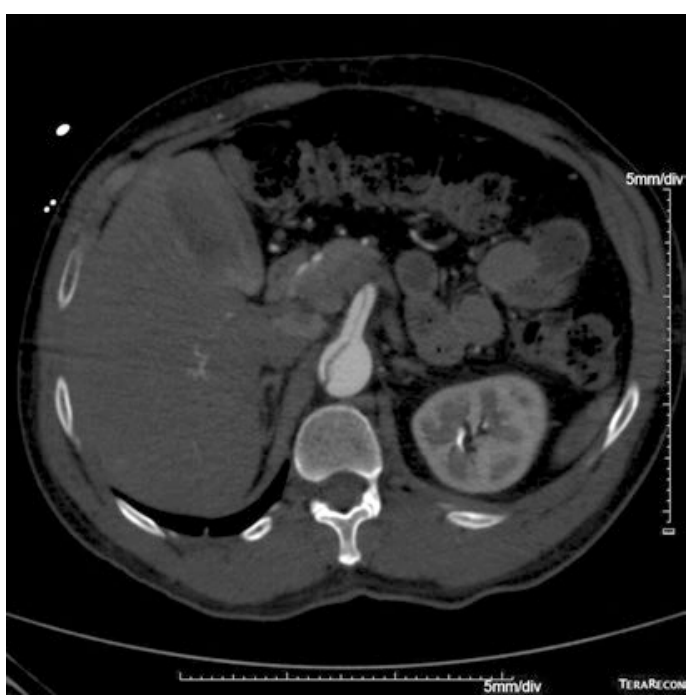

Fig. 30.2 Progression of the false lumen into the superior mesenteric artery with distal outflow and maintained perfusion of both true and false lumen

- Progression of the false lumen into a branch (eccentric or circumferential) without distal re-entry resulting in an extrinsic compression of the true lumen and various degrees of malperfusion (from flow reduction to complete obstruction of both lumina) (Fig. 30.3a-c)

- Ostial disconnection with or without flowlimiting intimal tears

The understanding of these mechanisms is crucial to identify the right strategy to resolve a possible malperfusion, hence the importance of imaging modalities and their interpretation.

\subsection{The Role of Imaging}

The term malperfusion-as opposed to hypoperfusion-suggests a lack of adequate blood supply; however it is commonly used both in the context of hypoperfusion with and without end-organ ischaemia. This distinction is relevant both in terms of terminology and for clinical decision-making as the end-organ ischaemia and not the malperfusion alone (usually based on radiological findings) that defines the need for intervention.
Imaging plays a critical role in suggesting a visceral malperfusion and in defining the mechanism of the malperfusion; it has however significant limitations when it comes to differentiating between hypoperfusion and end-organ ischaemia.

CT tomography, MR and ultrasound imaging all play an important role in the imaging of aortic dissection [9, 14-16].

\subsubsection{Computed Tomography}

In the acute setting, computed tomography remains the imaging of choice for the diagnosis and characterisation of aortic dissection.

CT angiography is widely available in the emergency setting and combines a high spatial resolution with a rapid acquisition time. Sequences in the arterial phase and venous phase can allow a reasonably good description of both the true and the false lumen and their relative perfusion. Several factors however can influence the quality of the imaging and the enhancement of both lumina and the aortic branches. Those factors include heart rate, ejection fraction, blood pressure and movement artefacts. All these factors are particularly relevant in the diagnosis of visceral malperfusion (in setting of an acute aortic dissection). The right timing of the acquisition can be difficult to predict, and this can influence significantly the enhancement of true and false lumen. In most cases CT angiography is accurate in demonstrating the involvement of the ostia of the main visceral arteries and in differentiation between different mechanisms of obstruction. Triple-phase CT has improved our ability to assess flow and thrombosis in the true and false lumen and should be considered the protocol of choice in most instances. ECG-gated protocols have proven to be useful to reduce misinterpretations due to motion artefact and more importantly to characterise intimal flap motion although its use is somehow limited by the reduced field of acquisition and their role in the diagnosis of visceral branch malperfusion still has to be demonstrated [16-18]. 

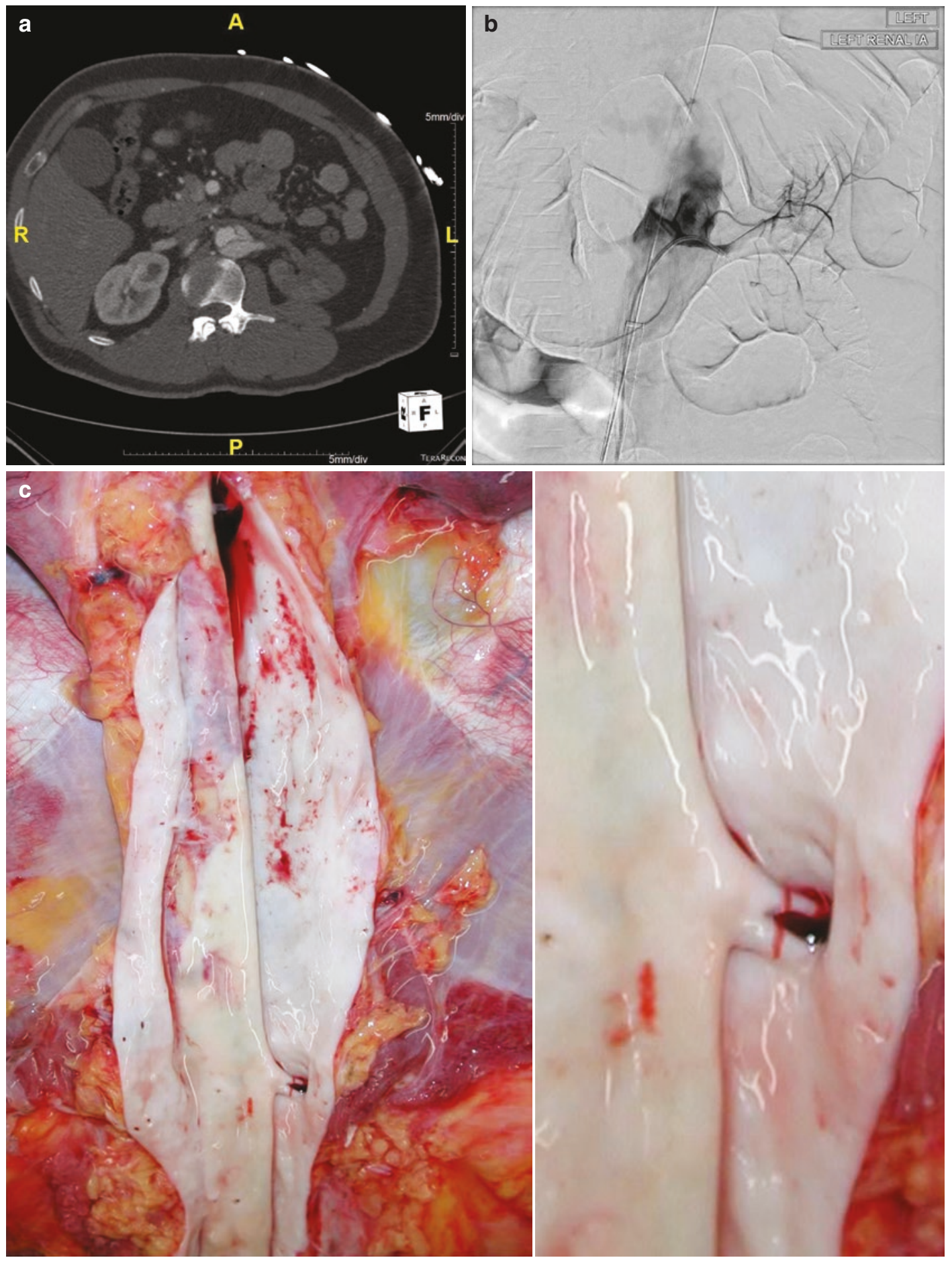

Fig. 30.3 (a, b) Progression of false lumen into the left renal artery with no distal re-entry with compression of the true lumen and organ malperfusion. (c) Autoptic findings of progression of the false lumen into the left renal artery (Courtesy of Chiesa/Tshomba et al., San Raffaele Hospital, Milan) 


\subsubsection{MR Imaging}

MR has an increasingly important role, both in the acute and in the chronic phase, for its potential of providing simultaneously morphological and functional information and for the absence of ionising radiations [14]. In the acute setting the main limitations remain availability and acquisition time. The most commonly used modality is MR angiogram. First-pass MRI is commonly used to identify false lumen thrombosis. However it is important to remember that both CT angiography and first-pass MRI have been shown to overestimate the amount of false lumen thrombosis by up to sixfold when compared to more advanced although not commonly available MRI protocols [19], hence the need for a high degree of circumspection when interpreting those investigations [16]. MR is a fastevolving technology, and it will certainly play an increasingly important role in acute aortic syndromes [20].

\subsubsection{Ultrasound Modalities}

Ultrasound modalities have a somehow limited but important role in the diagnosis of aortic dissections complicated by visceral malperfusion.

Colour Doppler ultrasonography can be extremely helpful to evaluate a haemodynamic compromise at the level of the renal and superior mesenteric artery [9, 21-23]. Limitations intrinsic to the technique are represented by the availability of experienced operators in an urgent setting, the morphology and the clinical and haemodynamic status of the patient. Intravascular ultrasound (IVUS) is mainly used intraoperatively to define the anatomy and the interaction between true and false lumen and to guide precise treatment both at the level of the aorta and its branches [24].

Transoesophageal ultrasound can demonstrate the haemodynamic conflict between true and false lumen helping to rule out dynamic, intermittent compressions of the true lumen at the level of the descending thoracic aorta (Fig. 30.4a, b).
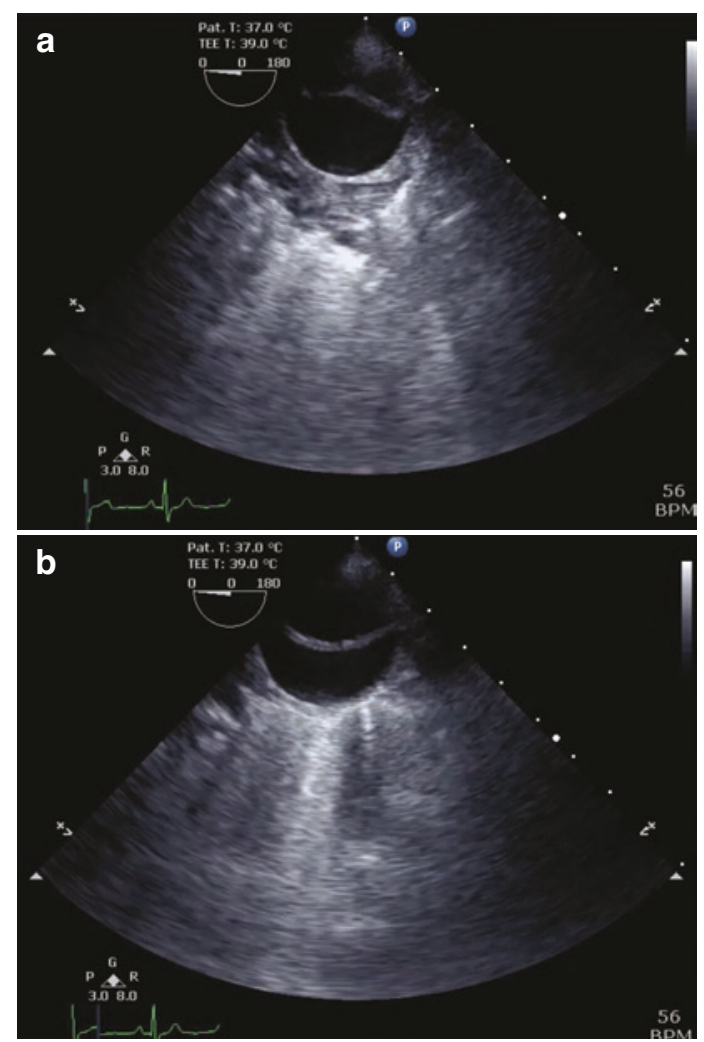

Fig. 30.4 (a) Dynamic compression of the true lumen during the systolic phase on TEE. (b) Distension of the true lumen during the diastolic phase on TEE

\subsubsection{Limitations and Perspective in Imaging of Renal Malperfusion}

In case of involvement of the renal arteries, it is a common finding to observe a defect or delay of enhancement of one or-rarely-both kidneys. Given the above-mentioned limitation and the rapid acquisition, it is somehow difficult to give a functional interpretation to these findings as the organ can still be well perfused despite a poor enhancement. While renal perfusion scintigraphy might have a role in the subacute and chronic phase, interesting perspectives are suggested by preliminary studies investigating the use of CT perfusion scanning for renal malperfusion [25]. In a report from Johns Hopkins Hospital on 38 patients, renal malperfusion could be accurately predicted by end-diastolic 
velocity (EDV) measurements on duplex ultrasound imaging [26]. IVUS and selective pressure measurements have been used successfully to define and guide treatment for renal malperfusion; however, there is no clear description on the technique to measure pressure gradients (whether, e.g. it is measured in the aorta or in the target vessel), and specific advantages with the use of IVUS assessment as opposed to standard imaging with CTA and intraoperative angiography still have to be demonstrated [27].

\subsubsection{Limitations and Perspectives in Imaging of Mesenteric Malperfusion}

Radiological diagnosis of mesenteric malperfusion is still extremely difficult.

Multiphase CT (usually biphasic is recommended, but in the context of an acute dissection, a triple-phase CT should be considered the gold standard) remains the imaging of choice. CT findings have been associated with advanced and in most cases irreversible mesenteric ischaemia and include intestinal dilatation and thickness, reduction or absence of visceral enhancement, pneumatosis intestinalis and portal venous gas and free intraperitoneal air [28]. On the other hand, improvement in CT protocols and interpretation are showing promising results for the diagnosis of intermediate grades of ischaemia [29]. Of note no other imaging modalities are currently recommended by international guideline on management of acute mesenteric ischaemia [30, 31]; nevertheless there is evidence and hope that different imaging modalities, namely, MRI, will be in the future validated and will become an additional tool in the diagnosis of mesenteric ischaemia [32, 33].

\subsection{Recognition and Management of Visceral Malperfusion}

Distinction between hypoperfusion and malperfusion is often difficult as malperfusion does not automatically translate in end-organ ischaemia.
Signs and symptoms can be difficult to interpret; however, clinical assessment of the patient remains key for the diagnosis and for decisionmaking particular when there is discrepancy between clinical and radiological findings.

Operative treatments whether this translates in open or endovascular interventions carry significant risks and should be justified on clinical ground.

Although surgical aortic replacement remains the gold standard in management of type A aortic dissections [9], there is increasing interest and awareness regarding the potential role of malperfusion first strategies that combine endovascular and open surgical techniques to improve outcomes in patient presenting with visceral malperfusion and stable type A dissections [2].

Endovascular stent grafting is widely recognised as the treatment of choice in acute complicated type B dissections, and endovascular techniques should be favoured as the first choice in the management of visceral malperfusion in most cases [34-37]. Open surgical repair for visceral malperfusion in aortic dissection requires experience and can be considered in selected patients and high-volume centres for this kind of surgery [37].

Understanding the mechanism of obstruction plays an important role for the choice of the most appropriate from of intervention. Dynamic obstruction will almost invariably need a central aortic treatment aiming at covering the proximal entry tear and re-equilibrate the perfusion pressure between true and false lumen, while static obstructions of the visceral branches might benefit of more focused treatment that can include selective stenting and fenestration techniques, and combined mechanisms of dynamic and static obstructions conversely need a combined approach.

There are however not large series or highquality literature reporting combined approaches and strategies detailing the suspected mechanisms of malperfusion; aortic treatment has been adopted as the first-line strategy in a large centre, while on the other hand, there are relatively small series reporting fenestration techniques as the main treatment for visceral malperfusion [27, $34,38,39]$. 
It is of extreme interest to notice that multiple reports failed to show an advantage in survival for patients who underwent branch vessel stenting or any intervention for malperfusion with particular regard to renal malperfusion raising the question whether renal malperfusion alone (particularly when it comes to a single kidney malperfusion) should be an indication at all for any kind of treatment [26, 38].

\subsubsection{A Note on Blood Pressure Management in Acute Aortic Dissections Complicated by Visceral Malperfusion: Is There a Role for Permissive Hypertension in the Presence of Malperfusion?}

Aggressive blood pressure management has an established role in the treatment of acute aortic dissections for prevention of progression of the dissection and rupture of the aorta [39, 40].

However there is some evidence that higher blood perfusion pressure might be beneficial in patients with visceral malperfusion [38]. High mean arterial pressures (MAP) are also often adopted as a standard practice in the management of acute dissections complicated pre- or postoperatively by spinal cord ischaemia. Despite convincing data supporting this practice [41], it is interesting to highlight that in traumatic spinal cord injuries, no advantages have been demonstrated with arbitrarily set high MAP goals [42], and overall there is limited evidence to support it.

Overall there is certainly scope to further investigate the role of permissive hypertension in the acute setting of aortic dissections complicated by malperfusion.

\subsection{Challenges of Renal Malperfusion}

A degree of renal failure is very common in the setting of acute aortic dissections. The aetiology of possible acute kidney injury in this context is most of the times the results of a multifactorial insult to the kidney [9]. Regardless of concomitant findings of delayed perfusion of the kidney with evidence of dynamic or static compression (usually this inflects in a picture of true lumen compression causing haemodynamic compression and flow reduction associated or less to extension of the dissection into the lumen of the artery), a state of shock, possible dehydration, aggressive intravenous blood pressure control and multiple imaging with contrast can all contribute to an acute kidney injury even in an otherwise well-perused organ. In most cases a reduced enhancement on CT imaging of a kidney will be interpreted as a sign of malperfusion of the organ. However the distinction between delayed perfusion, malperfusion and end-organ ischaemia is not only an academic exercise as it affects the decision-making and possibly the prognosis of the patient. CT findings however are known to be unreliable as they depend on the haemodynamic in and between the two lumina, and even with multiple-phase CT or gated imaging, they should be interpreted with caution [16, 19]. More subtle interpretation of the CT (as kidney size or presence of contrast in a delayed phase) can still give testimony of the perfusion of the kidney despite poor enhancement.

When it comes to indications to treatment, renal failure is a well-known predictor of poor prognosis in cardiovascular interventions as well as in acute aortic dissections [9]. This usually leads to the somehow logical temptation of aggressively correcting any reversible cause of it. Different considerations though seem to be necessary when it comes to chronic kidney disease (CKD) and acute kidney injuries in the setting of aortic dissection with or without underlying CKD.

Chronic kidney disease can be a marker of a more advanced form of atheromasic disease and/ or underlying comorbidities (diabetes) as this can often explain its importance as a prognostic factor. Similarly in the setting of acute aortic dissection, a renal malperfusion could be the marker of a more severe form of dissection, hence its relationship with poorer outcomes.

Overall aggressive revascularisation strategies based on the presence of kidney malperfusion and a biochemical picture of renal failure could be a 
complete inappropriate approach, and it should be carefully weighted with the complexity of the required/proposed treatment.

On the other hand, a possible rationale for treatment even in the case of a single renal malperfusion could be a refractory hypertension [as mentioned before though that there is some evidence that hypertension might be linked to better outcome in the presence of a malperfusion syndrome [38]].

\subsection{Challenges of Mesenteric Malperfusion}

Mesenteric malperfusion is the more insidious and serious form of malperfusion, and overall it is associated with a poor prognosis in the setting of acute aortic dissection [4, 7].

As discussed previously the instrumental diagnosis is often difficult, and often only irreversible ischaemia is clearly demonstrated on imaging.

On the other hand, available biomarkers have failed to demonstrate high sensitivity and specificity. As stated in the guidelines of the World Society of Emergency Surgery, "There are no laboratory studies that are sufficiently accurate to identify the presence or absence of ischemic or necrotic bowel, although elevated L-lactate, and D-dimer may assist," and this statement is supported by most of the available literature $[30,43,44]$. According to the European Society of Trauma and Emergency Surgery (ESTES), "Normal serum lactate level does not exclude acute mesenteric ischemia (AMI) and should not be used for diagnosis" [31].

Once mesenteric ischaemia has been demonstrated on clinical ground and with ominous signs on CT imaging, it will require in most of the cases immediate laparotomy and bowel resection more than any form of revascularisation.

As a result clinical diagnosis together with a suspicion of malperfusion on CT imaging should guide clinical practice. This is of course made even more difficult by confusing factor as could be sedation, analgesia and concomitant malperfusion of different aortic branches. Feasibility and safety of laparoscopy for early detection of bowel ischaemia has been demonstrated [45]; however there is insufficient evidence to support the routine use of laparoscopy in AMI [31].

Overall the early diagnosis of mesenteric malperfusion remains an unresolved challenge. In the next paragraph, we will expand further on emerging biomarkers that might radically change our practice in the future.

\subsection{Future Perspectives: Biomarkers and Early Diagnosis of Visceral Malperfusion}

Early recognition and diagnosis remain the greatest challenge and play a crucial role in improving our understanding and ultimately our management of acute aortic dissection complicated by visceral malperfusion.

The use of biomarkers, in combination with advanced imaging techniques and conventional biochemistry analysis, may provide a better way of assessing ongoing VMP following aortic dissection. A good biomarker should be easy to measure, have an earlier detectability than conventional assays (such as creatinine/lactate), be organ-specific identity type and preferably be able to predict the severity of malperfusion as well as allow for monitoring of any pharmacological/surgical responses to therapy. Theoretically, these biomarkers can be measured at the gene, RNA and protein (including cell surface, intracellular and circulating) level. It is generally the circulating proteins that are easier to measure, although techniques such a flow cytometry and polymerase chain reaction are now utilised relatively routinely in the clinic.

During the period of organ ischaemia, the function of the endothelial cell barrier is impaired [46], resulting in an increase in vascular permeability and leakage [47]. During this period, a range of changes occurs in the transcriptional control of gene expression. For example, oxygen-sensing prolyl hydroxylase (PHD) enzymes, which require oxygen as a cofactor, are inhibited which leads to the activation of hypoxia and 
inflammatory signalling cascades that control the stability of the transcription factors hypoxiainducible factor (HIF) and nuclear factor- $\kappa \mathrm{B}$

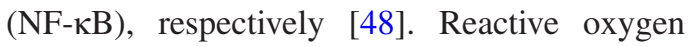
species (ROS) increased ischaemia and reperfusion [49] and are implicated in the tissue damage that occurs during this period by altering cellular ribonucleic acids (RNAs), lipids and proteins. Measurement of the redox state of patients may provide a way of assessing for VMR as well as predicting clinical outcome [50].

Tissue ischaemia also results in acute changes in cellular infiltrate within the circulation and organ itself. Studies have shown that T cell lymphocytes accumulate during organ ischaemia and reperfusion [51] and measurement of changes in $\mathrm{T}$ cell subsets and phenotype using flow cytometry may provide evidence of ongoing organ ischaemia. For example, further, IL-17 is produced by $\gamma \delta \mathrm{T}$ cells during ischaemia and reperfusion injury [52]. This sterile inflammation during ischaemia is also characterised by the recruitment of specific subsets of monocytes from the bone marrow and splenic reservoir to participate in healing [53, 54]. One disadvantage of these assays is that changes in cellular phenotype are not usually organ specific and further work is needed to determine specific changes in mononuclear cell populations during, for example, bowel, liver and renal ischaemia.

Despite its poor sensitivity and specificity, serum creatinine is still utilised as the indicator for renal injury/ischaemia. Cystatin $\mathrm{C}$ may be a more reliable biomarker of renal malperfusion, and more novel markers such as urinary $N$-acetyl$\beta$-D-glucosaminidase (NAG), kidney injury molecule-1 (KIM-1) and neutrophil gelatinaseassociated lipocalin (NGAL) have recently been shown to be sensitive, early markers of tubular damage in renal ischaemia [55]. There is increasing interest in the use of microRNAs (miRNAs), noncoding RNAs that post-transcriptionally regulate gene expression, for detecting organ-specific changes. These biomarkers are stable, have low interindividual variability and can be measured in samples of serum and plasma. For example, miR-30a, miR-30e and miR-188 are upregulated between two- and threefold [56] during kidney injury. Further research is needed into evaluating changes that occur in circulating miRNAs during aortic dissection and then correlating these with organ ischaemia.

The emerging use of "omics" in the discovery of biomarkers with the aid of mass spectrometry and bioinformatics has also recently provided new avenues in the monitoring of visceral malperfusion. Proteomic studies investigate the pool of full-length, truncated and post-transductionally modified proteins and peptides, whereas metabolomics explores the metabolic process of peptides, saccharides and lipids. Studies have utilised the omics approach for the assessment of biomarkers of gut malperfusion. The mature enterocyte expresses intestinal FABP (I-FABP), and urinary I-FABP has been shown to be a biomarker with high specificity and sensitivity for the diagnosis of acute intestinal ischaemia $[57,58]$. I-FABP is considered to be one of the most promising biomarkers for intestinal ischaemia along with D-dimer and a-GST [59]. The advantage of I-FABP above a-GST and D-dimer is, of course, its tissue specificity [60].

During liver ischaemia, activated Kupffer cells, liver tissue and distant organs secrete TNF- $\alpha$ [61] which can be measured in serum. Matrix metalloproteinases (MMPs), such as MMP-9, are detected in the serum of patients following liver ischaemia/reperfusion, and a range of MMPs, including MMP-2, MMP-3, MMP-8, MMP-10, MMP-12 and MMP-13, are upregulated during hepatic ischaemia [62]. However, neither TNF- $\alpha$ nor MMPs are liver specific as they can be increased during systemic inflammation, and further work is needed in biomarkers that can detect VMP due to reduced flow in the coeliac axis.

\section{References}

1. Gray H. Anatomy of the human body. 23rd ed. Philadelphia: Lea \& Febiger; 1936.

2. Kamman AV, Yang B, Kim KM, Williams DM, Michael Deeb G, Patel HJ. Visceral malperfusion in aortic dissection: the Michigan experience. Semin Thorac Cardiovasc Surg. 2017;29(2):173-8. 
3. Tanaka A, Estrera AL. Mesenteric malperfusion: the insidious, dreadful enemy. Semin Thorac Cardiovasc Surg. 2017;29(2):179-80.

4. Jonker FH, Patel HJ, Upchurch GR, Williams DM, Montgomery DG, Gleason TG, et al. Acute type B aortic dissection complicated by visceral ischemia. J Thorac Cardiovasc Surg. 2015;149(4):1081-6.e1.

5. Rampoldi V, Trimarchi S, Eagle KA, Nienaber CA, Oh JK, Bossone E, et al. Simple risk models to predict surgical mortality in acute type A aortic dissection: the International Registry of Acute Aortic Dissection score. Ann Thorac Surg. 2007;83(1):55-61.

6. Geirsson A, Szeto WY, Pochettino A, McGarvey ML, Keane MG, Woo YJ, et al. Significance of malperfusion syndromes prior to contemporary surgical repair for acute type A dissection: outcomes and need for additional revascularizations. Eur J Cardiothorac Surg. 2007;32(2):255-62.

7. Berretta P, Patel HJ, Gleason TG, Sundt TM, Myrmel T, Desai N, et al. IRAD experience on surgical type A acute dissection patients: results and predictors of mortality. Ann Cardiothorac Surg. 2016;5(4):346-51.

8. Di Eusanio M, Trimarchi S, Patel HJ, Hutchison S, Suzuki T, Peterson MD, et al. Clinical presentation, management, and short-term outcome of patients with type A acute dissection complicated by mesenteric malperfusion: observations from the International Registry of Acute Aortic Dissection. J Thorac Cardiovasc Surg. 2013;145(2):385-90.e1.

9. Crawford TC, Beaulieu RJ, Ehlert BA, Ratchford EV, Black JH 3rd. Malperfusion syndromes in aortic dissections. Vasc Med. 2016;21(3):264-73.

10. Goldberg JB, Lansman SL, Kai M, Tang GHL, Malekan R, Spielvogel D. Malperfusion in type A dissection: consider reperfusion first. Semin Thorac Cardiovasc Surg. 2017;29(2):181-5.

11. Tanaka K, Chikazawa G, Sakaguchi T, Totsugawa T, Tamura K, Yoshitaka H. Hybrid treatment for type A acute aortic dissection with multiorgan malperfusion. Ann Thorac Surg. 2014;98(3):1118-20.

12. Williams DM, Lee DY, Hamilton BH, Marx MV, Narasimham DL, Kazanjian SN, et al. The dissected aorta: part III. Anatomy and radiologic diagnosis of branch-vessel compromise. Radiology. 1997;203(1):37-44.

13. Midulla M, Fattori R, Beregi JP, Dake M, Rousseau H. Aortic dissection and malperfusion syndrome: a when, what and how-to guide. Radiol Med. 2013;118(1):74-88.

14. Moore AG, Eagle KA, Bruckman D, Moon BS, Malouf JF, Fattori R, et al. Choice of computed tomography, transesophageal echocardiography, magnetic resonance imaging, and aortography in acute aortic dissection: International Registry of Acute Aortic Dissection (IRAD). Am J Cardiol. 2002;89(10):1235-8

15. Baliga RR, Nienaber CA, Bossone E, Oh JK, Isselbacher EM, Sechtem U, et al. The role of imaging in aortic dissection and related syndromes. JACC Cardiovasc Imaging. 2014;7(4):406-24.
16. Donati T, Wilson J, Kolbel T, Clough RE. Modern diagnostics for type B aortic dissection. Gefässchirurgie. 2015;20(6):420-7.

17. Yang S, Li X, Chao B, Wu L, Cheng Z, Duan Y, et al. Abdominal aortic intimal flap motion characterization in acute aortic dissection: assessed with retrospective ECG-gated thoracoabdominal aorta dual-source CT angiography. PLoS One. 2014;9(2):e87664.

18. Ganten MK, Weber TF, von Tengg-Kobligk H, Bockler D, Stiller W, Geisbusch P, et al. Motion characterization of aortic wall and intimal flap by ECGgated CT in patients with chronic B-dissection. Eur J Radiol. 2009;72(1):146-53.

19. Clough RE, Hussain T, Uribe S, Greil GF, Razavi R, Taylor PR, et al. A new method for quantification of false lumen thrombosis in aortic dissection using magnetic resonance imaging and a blood pool contrast agent. J Vasc Surg. 2011;54(5):1251-8.

20. Wang GX, Hedgire SS, Le TQ, Sonis JD, Yun BJ, Lev MH, et al. MR angiography can guide ED management of suspected acute aortic dissection. Am J Emerg Med. 2017;35(4):527-30.

21. Catalini R, Alborino S, Giovagnoli A, Zingaretti O. Color Duplex evaluation of the mesenteric artery (). J Ultrasound. 2010;13(3):118-22.

22. Perko MJ. Duplex ultrasound for assessment of superior mesenteric artery blood flow. Eur J Vasc Endovasc Surg. 2001;21(2):106-17.

23. AbuRahma AF, Stone PA, Srivastava M, Dean LS, Keiffer T, Hass SM, et al. Mesenteric/celiac duplex ultrasound interpretation criteria revisited. J Vasc Surg. 2012;55(2):428-36.e6; discussion 35-6.

24. Koschyk DH, Meinertz T, Hofmann T, Kodolitsch YV, Dieckmann C, Wolf W, et al. Value of intravascular ultrasound for endovascular stent-graft placement in aortic dissection and aneurysm. J Card Surg. 2003;18(5):471-7.

25. Liu D, Liu J, Wen Z, Li Y, Sun Z, Xu Q, et al. 320row $\mathrm{CT}$ renal perfusion imaging in patients with aortic dissection: a preliminary study. PLoS One. 2017;12(2):e0171235.

26. Osgood MJ, Grimm J, Abularrage CJ, Lum YW, Call D, Black JH III. VESS24. Duplex ultrasound assessment and outcomes of renal malperfusion syndromes after acute aortic dissection. J Vasc Surg. 2017;65(6):29S.

27. Barnes DM, Williams DM, Dasika NL, Patel HJ, Weder AB, Stanley JC, et al. A single-center experience treating renal malperfusion after aortic dissection with central aortic fenestration and renal artery stenting. J Vasc Surg. 2008;47(5):903-10; discussion $10-1$.

28. Kirkpatrick ID, Kroeker MA, Greenberg HM. Biphasic CT with mesenteric CT angiography in the evaluation of acute mesenteric ischemia: initial experience. Radiology. 2003;229(1):91-8.

29. Moschetta M, Telegrafo M, Rella L, Stabile Ianora AA, Angelelli G. Multi-detector CT features of acute intestinal ischemia and their prognostic correlations. World J Radiol. 2014;6(5):130-8. 
30. Bala M, Kashuk J, Moore EE, Kluger Y, Biffl W, Gomes CA, et al. Acute mesenteric ischemia: guidelines of the World Society of Emergency Surgery. World J Emerg Surg. 2017;12:38.

31. Tilsed JVT, Casamassima A, Kurihara H, Mariani D, Martinez I, Pereira J, et al. ESTES guidelines: acute mesenteric ischaemia. Eur J Trauma Emerg Surg. 2016;42:253-70.

32. Mazzei MA, Guerrini S, Cioffi Squitieri N, Imbriaco G, Chieca R, Civitelli S, et al. Magnetic resonance imaging: is there a role in clinical management for acute ischemic colitis? World J Gastroenterol. 2013;19(8):1256-63.

33. Saba L, Berritto D, Iacobellis F, Scaglione M, Castaldo S, Cozzolino S, et al. Acute arterial mesenteric ischemia and reperfusion: macroscopic and MRI findings, preliminary report. World J Gastroenterol. 2013;19(40):6825-33.

34. Lauterbach SR, Cambria RP, Brewster DC, Gertler JP, Lamuraglia GM, Isselbacher EM, et al. Contemporary management of aortic branch compromise resulting from acute aortic dissection. J Vasc Surg. 2001;33(6):1185-92.

35. White RA, Miller DC, Criado FJ, Dake MD, Diethrich $\mathrm{EB}$, Greenberg RK, et al. Report on the results of thoracic endovascular aortic repair for acute, complicated, type $\mathrm{B}$ aortic dissection at 30 days and 1 year from a multidisciplinary subcommittee of the Society for Vascular Surgery Outcomes Committee. J Vasc Surg. 2011;53(4):1082-90.

36. Nienaber CA, Fattori R, Lund G, Dieckmann C, Wolf $\mathrm{W}$, von Kodolitsch Y, et al. Nonsurgical reconstruction of thoracic aortic dissection by stent-graft placement. N Engl J Med. 1999;340(20):1539-45.

37. Szeberin Z, Dosa E, Fehervari M, Csobay-Novak C, Pinter N, Entz L. Early and long-term outcome after open surgical suprarenal aortic fenestration in patients with complicated acute type B aortic dissection. Eur J Vasc Endovasc Surg. 2015;50(1):44-50.

38. Ryan C, Vargas L, Mastracci T, Srivastava S, Eagleton M, Kelso R, et al. Progress in management of malperfusion syndrome from type B dissections. J Vasc Surg. 2013;57(5):1283-90; discussion 90.

39. Mokashi SA, Svensson LG. Guidelines for the management of thoracic aortic disease in 2017. Gen Thorac Cardiovasc Surg. 2017.

40. Writing C, Riambau V, Bockler D, Brunkwall J, Cao $\mathrm{P}$, Chiesa R, et al. Editor's choice-Management of descending thoracic aorta diseases: clinical practice guidelines of the European Society for Vascular Surgery (ESVS). Eur J Vasc Endovasc Surg. 2017;53(1):4-52.

41. Kise Y, Kuniyoshi Y, Inafuku H, Nagano T, Hirayasu T, Yamashiro S. Directly measuring spinal cord blood flow and spinal cord perfusion pressure via the collateral network: correlations with changes in systemic blood pressure. J Thorac Cardiovasc Surg. 2015;149(1):360-6.

42. Martin ND, Kepler C, Zubair M, Sayadipour A, Cohen M, Weinstein M. Increased mean arterial pres- sure goals after spinal cord injury and functional outcome. J Emerg Trauma Shock. 2015;8(2):94-8.

43. Acosta S, Nilsson T. Current status on plasma biomarkers for acute mesenteric ischemia. J Thromb Thrombolysis. 2012;33(4):355-61.

44. Ambe PC, Kang K, Papadakis M, Zirngibl H. Can the preoperative serum lactate level predict the extent of bowel ischemia in patients presenting to the emergency department with acute mesenteric ischemia? Biomed Res Int. 2017;2017:8038796.

45. Tshomba Y, Coppi G, Marone EM, Bertoglio L, Kahlberg A, Carlucci M, et al. Diagnostic laparoscopy for early detection of acute mesenteric ischaemia in patients with aortic dissection. Eur J Vasc Endovasc Surg. 2012;43(6):690-7.

46. Ogawa S, Gerlach H, Esposito C, Pasagian-Macaulay A, Brett J, Stern D. Hypoxia modulates the barrier and coagulant function of cultured bovine endothelium. Increased monolayer permeability and induction of procoagulant properties. J Clin Invest. 1990;85(4):1090-8.

47. Ogawa S, Koga S, Kuwabara K, Brett J, Morrow B, Morris SA, et al. Hypoxia-induced increased permeability of endothelial monolayers occurs through lowering of cellular cAMP levels. Am J Phys. 1992;262(3 Pt 1):C546-54.

48. Eltzschig HK, Carmeliet P. Hypoxia and inflammation. N Engl J Med. 2011;364(7):656-65.

49. Iadecola C, Anrather J. The immunology of stroke: from mechanisms to translation. Nat Med. 2011;17(7):796-808.

50. Paspalj D, Nikic P, Savic M, Djuric D, Simanic I, Zivkovic V, et al. Redox status in acute ischemic stroke: correlation with clinical outcome. Mol Cell Biochem. 2015;406(1-2):75-81.

51. Schroeter M, Jander S, Witte OW, Stoll G. Local immune responses in the rat cerebral cortex after middle cerebral artery occlusion. J Neuroimmunol. 1994;55(2):195-203.

52. Shichita T, Sugiyama Y, Ooboshi H, Sugimori H, Nakagawa R, Takada I, et al. Pivotal role of cerebral interleukin-17-producing gammadeltaT cells in the delayed phase of ischemic brain injury. Nat Med. 2009;15(8):946-50.

53. Swirski FK, Nahrendorf M, Etzrodt M, Wildgruber M, Cortez-Retamozo V, Panizzi P, et al. Identification of splenic reservoir monocytes and their deployment to inflammatory sites. Science. 2009;325(5940):612-6.

54. Patel AS, Smith A, Nucera S, Biziato D, Saha P, Attia $\mathrm{RQ}$, et al. TIE2-expressing monocytes/macrophages regulate revascularization of the ischemic limb. EMBO Mol Med. 2013;5(6):858-69.

55. Andreucci M, Faga T, Pisani A, Perticone M, Michael A. The ischemic/nephrotoxic acute kidney injury and the use of renal biomarkers in clinical practice. Eur $\mathrm{J}$ Intern Med. 2017;39:1-8.

56. Sun SQ, Zhang T, Ding D, Zhang WF, Wang XL, Sun $\mathrm{Z}$, et al. Circulating microRNA-188, -30a, and $-30 \mathrm{e}$ as early biomarkers for contrast-induced acute kidney injury. J Am Heart Assoc. 2016;5(8):e004138. 
57. Salim SY, Young PY, Churchill TA, Khadaroo RG. Urine intestinal fatty acid-binding protein predicts acute mesenteric ischemia in patients. J Surg Res. 2017;209:258-65.

58. Sun DL, Cen YY, Li SM, Li WM, Lu QP, Xu PY. Accuracy of the serum intestinal fatty-acidbinding protein for diagnosis of acute intestinal ischemia: a meta-analysis. Sci Rep. 2016;6:34371.

59. Derikx JP, Schellekens DH, Acosta S. Serological markers for human intestinal ischemia: a systematic review. Best Pract Res Clin Gastroenterol. 2017;31(1):69-74.

60. Pelsers MM, Namiot Z, Kisielewski W, Namiot A, Januszkiewicz M, Hermens WT, et al. Intestinal- type and liver-type fatty acid-binding protein in the intestine. Tissue distribution and clinical utility. Clin Biochem. 2003;36(7):529-35.

61. Peralta C, Fernandez L, Panes J, Prats N, Sans M, Pique JM, et al. Preconditioning protects against systemic disorders associated with hepatic ischemiareperfusion through blockade of tumor necrosis factor-induced P-selectin up-regulation in the rat. Hepatology. 2001;33(1):100-13.

62. Hamada T, Duarte S, Tsuchihashi S, Busuttil RW, Coito AJ. Inducible nitric oxide synthase deficiency impairs matrix metalloproteinase- 9 activity and disrupts leukocyte migration in hepatic ischemia/reperfusion injury. Am J Pathol. 2009;174(6):2265-77. 